\title{
ADAPTIVE CONTROL OF RIGID ROBOTS WITH UNCERTAIN DRIVE GAINS USING PARAMETER UNFOLDING
}

\author{
RAUL GUENTHER and LIU HSU* \\ Department of Mechanical Engineering - UFSC \\ Caixa Postal 476 - 88035/460 - Florianópolis - SC \\ e-mail: emc1rag@ufsc.br \\ *Department of Electrical Engineering - COPPE/UFRJ
}

\begin{abstract}
Significant progress in the analysis and design of adaptive tracking control schemes for rigid robot manipulators was achieved in the last decade [5]. Many of these schemes have been designed at the torque input level. That is, the dynamics associated with the actuators is neglected. Moreover to design adaptive control at the torque input level one needs to assume that the motor drive gains are exactly known. Then, in the case of electrically-driven robot manipulators, the gear transmission ratios, the amplifier gains, the motor torque constants and the electrical resistance of the motor armature circuit must be identified by using classical electrical machine techniques, or generally the constructors data are often used.
\end{abstract}

\section{INTRODUCTION}

Recently it has been demonstrated [1] that considerable variation occurs in experimental values obtained for the motor drive gains in case of an electrically-driven robot.

To avoid the necessity of exactly knowledge of these parameters new method was proposed in [4], to identify the dynamic model directly as a function of the motor currents.

In this paper we assume that the drive gains are not exactly available. As will be seen the effect of the motor drive gain uncertainty can be regarded as an input disturbance which could cause instability or residual errors.

In order to obtain global stability in sense of Lyapunov, and asymptotically convergence of the tracking errors, we propose the modified adaptive control scheme based on a technique introduced in [3], which we denominate "parameter unfolding". It consists in introducing an augmented regressor matrix and a corresponding augmented parameter vector in the adaptation law. The overparametrization is simple to obtain and can be seen as the unfolding of the adaptive parameter vector of the standart case (known gains), in $\mathrm{n}$ similar parameter vectors. The uncertain drive gain parameters can then be absorbed by a new estimated parameter vector.

This approach can be applied to robots with any type of actuator in which the drive gains are uncertain parameters. 


\section{2 - THE ROBOT MANIPULATOR MODEL}

Consider an n-link rigid manipulator with revolute joints. The familiar model is [10]

$$
[D(q)+J] \ddot{q}+C(q, \dot{q}) \dot{q}+g(q)=u
$$

where $\mathbf{q}(\mathbf{t})$ is the joint angular position vector: $\mathbf{D}(\mathbf{q})$ is the link inertia matrix; $\mathbf{J}$ is the rotor inertia matrix; $\mathbf{C} \dot{\mathbf{q}}$ is the vector of centripetal and Coriolis terms; $\mathbf{g}$ is the gravity torque vector and $u(t)$ is the torque vector.

In general the torque $\mathbf{u}(\mathbf{t})$ is not applied directly. For example. for electric motor drives, $u(t)$ is the torque response of drives to the input voltages. Thus either the drive gains or some more detailed dynamic model of the drive have to be accounted. Considering only the drive gains, expression (1) results

$$
[D(q)+J] \ddot{q}+C(q, \dot{q}) \dot{q}+g(q)=K v
$$

where $K$ is the diagonal motor drive gains matrix and $v(t)$ is the control vector (eg., voltages).

Remark 2.1 - In the case of permanent magnet DC motor actuators, $K=K_{m} R^{-1}$, where $K_{m}$ is the torque constant diagonal matrix, $\mathbf{R}$ is the electric resistance diagonal matrix and $v(t)$ is the motor terminal voltage vector.

\section{3 - ADAPTIVE TORQUE CONTROL WITR UNCERTAIN DRIVE MOTOR GAIN}

In adaptive tracking control schemes designed at the torque input level, we compute the torque u(t) to achieve tracking and then control the manipulator applying the control vector

$$
v=K_{0}^{-1} \mathbf{u}
$$


where $K_{0}$ is the nominal motor drive gains matrix. By combining (2) and (3) we obtain

$$
[D(q)+J] \ddot{q}+C(q, \dot{q}) \dot{q}+g(q)=\bar{K} \mathbf{u}
$$

where $\bar{K}=K_{0}^{-1}$ and $\Delta K:=\bar{K}-1$ is the uncertainty in the motor drive gain matrix ( $I$ is the nxn identity matrix).

Remark 3.1 - In the usual approaches the drive gains are considered exactly known and thus $K_{0}=K, \Delta K=0$ and $\bar{K}=1$. In this case (4) reduces trivialy to (1).

Consider that $u(t)$ is generated according to the Slotine-Li's adaptive torque control law [7]

$$
u=\left[D^{\prime}(q)+J^{\prime}\right] \ddot{q}_{r}+C^{\prime}(q, \dot{q}) \dot{q}_{r}+g^{\prime}(q)-K_{D} s
$$

where $D^{\prime}, J^{\prime}, C^{\prime}$ and $g^{\prime}$ represent respectively the terms $D, J, C$ and $g$ of (1) with estimated parameters, $K_{D}=K_{D}^{\top}>0$ is a diagonal design matrix, and

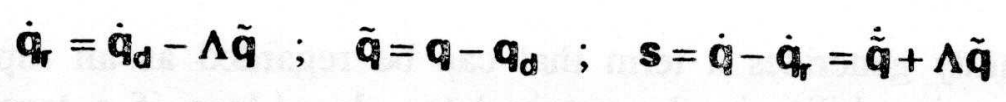

where $q_{d}$ is the desired angular joint position, $s$ is an reference error and $\Lambda$ is a positive diagonal matrix. According [7] (5) can be rewritten in the form

$$
u=\mathbf{Y}\left(\mathbf{q}, \dot{q}, \dot{q}_{r}, \ddot{q}_{r}\right) \theta^{\prime}-K_{D} s
$$

where $\mathbf{Y}\left(\mathbf{q}, \dot{\mathbf{q}}_{,} \dot{\mathbf{q}}_{r}, \ddot{q}_{r}\right)$ is an nxr matrix of known functions called regressor matrix, and $\theta^{\prime}$ is the nx 1 vector of estimated parameters. (7), i.e.,

Denote by $\theta$ the true parameter vector corresponding to the parametrization (5)-(6)-
i.e.

$$
[D(q)+J] \ddot{q}_{r}+c(q, \dot{q}) \dot{q}_{r}+g(q)=Y \theta
$$

Substituting (7) and (8) in (4) we can write the error equation 


$$
[D(q)+J] \dot{s}+\left[C(q, \dot{q})+\bar{K} K_{D}\right] s=\bar{K} Y \tilde{\theta}+\Delta K Y \theta
$$

where $\tilde{\theta}:=\theta^{\prime}-\theta$ is the parameter error.

The term $\Delta K Y \theta$ in (9) may be regarded as an input disturbance, and so this closedloop error system is similar to that obtained in $|6|$ where is shown that with the "gradient" adaptation law

$$
\dot{\theta}^{\prime}=-\Gamma Y^{\top} \mathbf{s}
$$

the closed-loop system (4)-(7)-(10) may result unstable. Hence, a robustness improvement of the adaptive control is necessary. Stability is recovered by employing a switching parameter $\sigma\left(\theta^{\prime}\right)$ in the adaptation law (10). For bounded disturbances, it can be proved $|6|$ that the filtered error $S$ converges to a residual set $\rho$ depending on the disturbance bound. Then, the tracking errors also converge to residual sets (see (6)).

Remark 3.2 - If $K_{o}=K$, there is no uncertainty in the motor drive gains and $\bar{K}=\mathbf{I}$. In this case the disturbance is zero and the tracking errors $\tilde{\mathbf{q}}(\mathbf{t}) \rightarrow 0$ and $\dot{\tilde{q}}(\mathbf{t}) \rightarrow 0$ as $\mathbf{t} \rightarrow \infty$ [7].

From the above it can be outlined that

1 - The drive gain uncertainty generates a term that can be regarded as an input disturbance which may cause instability in the manipulator closed-loop if a torque control with only gradient adaptation law is employed.

2 - Stability may be recovered by introducing a switching $\sigma$-modification factor in the adaptation law if the input disturbance is bounded. This unfortunately is not the present case since $\Delta K Y \theta$ is not bounded 'a priori'.

3 - The tracking errors depend on the uncertainty in the drive gains, the regressor matrix, the true parameter vector and of design parameters, namely the gain matrix $\Lambda$ $(\sec (6))$.

In this paper we propose the parameter unfolding to modify the adaptive control law. Then, global stability and global convergence to the tracking errors $\tilde{\mathbf{q}}(\mathbf{t})$ and $\dot{\tilde{\mathbf{q}}}(\mathbf{t})$ to zero can be guaranteed, even in the unknown drive gain case. 


\section{4 - ADAPTIVE CONTROL WITH PARAMETER UNFOLDING}

To achieve global stability and asymptotic convergence of the tracking errors, we propose a modified scheme in order to absorb the drive gains $K$ in the estimated parameter vector.

In this paper we adopt the Slotine-Li's adaptive control scheme $|7|$ to generate torque signals. It should be noted that the following approach can also be used with other adaptive control schemes.

Consider that $u(8)$ is generated as in (7). From (2) and (7)

$$
v=K^{-4} Y \theta^{\prime}-\bar{K}_{D} s \quad\left(\bar{K}_{D}:=K^{-1} K_{D}\right)
$$

Then, the feedforward part of $v$ is $V_{\mathbb{N}}=K^{-1} Y \theta^{\prime}$. We wish embed the drive gains $K$ in the estimated parameter vector. To do this let $Y$ be written as $Y^{T}=\left[\begin{array}{llll}y_{1} & y_{2} & \cdots & y_{n}\end{array}\right]$, where $y_{1}^{T}$ is the $i$-th row of $Y$. Now introduce the augmented regressor matrix $\mathrm{Y}_{\mathrm{a}}$ and a corresponding augmented parameter vector $\theta_{\mathrm{a}}^{\prime}$ as follows

$$
\begin{aligned}
& \mathbf{Y}_{\mathrm{a}}=\left[\begin{array}{ccccc}
\mathbf{y}_{1}{ }^{\top} & 0 & . & 0 & 0 \\
0 & \mathbf{y}_{2}{ }^{\top} & \cdot & 0 & 0 \\
\cdot & \cdot & \cdot & \cdot & \cdot \\
\cdot & \cdot & \cdot & \cdot & \cdot \\
0 & 0 & \cdot & 0 & \mathbf{y}_{\mathrm{n}}{ }^{\top}
\end{array}\right] ; \quad \theta_{\mathrm{a}}^{\prime}=\left[\begin{array}{c}
\theta_{\mathrm{a} 1}^{\prime} \\
\theta_{\mathrm{a} 2}^{\prime} \\
\cdot \\
\cdot \\
\theta_{\mathrm{an}}^{\prime}
\end{array}\right] \\
& \theta_{\mathrm{ai}}^{\prime \top}=\left[\begin{array}{llll}
\theta_{\mathrm{a} 11}^{\prime} & \theta_{\mathrm{a} 2}^{\prime} & \cdots & \theta_{\mathrm{alr}}^{\prime}
\end{array}\right]: \quad \mathrm{i}=1, \ldots \mathrm{n}
\end{aligned}
$$

Here is important to stress that the elements of $\theta_{a}^{\prime}$ will be regarded, in what follows, as independent estimated parameters. This means that overparametrization is being used and it simply generated by "unfolding" the original parameter vector $\theta^{\prime}$. This motivates the denomination "parameter unfolding".

Now one can write

$$
V_{n}=K^{-1} Y_{a} \theta_{a}^{\prime}=Y_{a} K_{a}^{-1} \theta_{a}^{\prime}: \quad K_{a}=\operatorname{diag}\left[K_{1} I_{r}\right]
$$


where $\mathbf{K}_{\mathbf{i}}$ is the $\mathbf{i}$-th diagonal element of $\mathbf{K}$, and $\mathbf{I}_{\mathbf{r}}$ is the rxr identity matrix. Note the important fact that the diagonal matrix $K^{-1}$ is to the left of $Y_{a}$, whereas the augmented matrix $K_{a}^{-1}$ is to the right of $Y_{a}$. The drive gains can therefore be absorbed by a new estimated parameter vector, namely,

$$
\hat{\theta}_{\mathbf{a}}=K_{\mathbf{a}}^{-1} \theta_{\mathbf{a}}^{\prime}
$$

The control vector is therefore given by (see (11),(14) and (15))

$$
\mathbf{v}=\mathbf{Y}_{\mathrm{a}} \hat{\theta}_{\mathrm{a}}-\overline{\mathbf{K}}_{\mathrm{D}} \mathbf{s}
$$

and the torque vector $u(t)$ in (1) results

$$
\mathbf{u}=\mathbf{K v}=K \mathbf{Y}_{\mathbf{a}} \hat{\theta}_{\mathbf{a}}-K_{\mathrm{D}} s=\mathbf{Y}_{\mathbf{a}} K_{\mathbf{a}} \hat{\theta}_{\mathbf{a}}-K_{\mathrm{D}} s
$$

The true parameter vector is $\theta$ (see(8)), and the corresponding true augmented parameter vector can be given by $\theta_{\mathrm{a}}^{\top}=\left[\begin{array}{lll}\theta^{\top} & \theta^{\top} \text {. . } \theta^{\boldsymbol{\top}}\end{array}\right]$. Correspondingly, the true

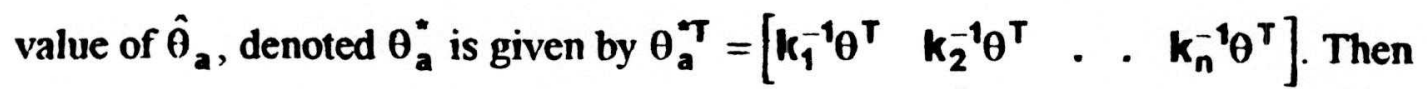

$$
\mathbf{Y} \theta=\mathbf{Y}_{\mathbf{a}} \mathbf{K}_{\mathbf{a}} \mathbf{K}_{\mathbf{a}}^{-1} \boldsymbol{\theta}_{\mathbf{a}}=\mathbf{Y}_{\mathbf{a}} \mathbf{K}_{\mathbf{a}} \theta_{\mathbf{a}}^{*}
$$

Substituting (17) and (18) in (1) we can write the error equation

$$
[D(q)+J] \dot{s}+\left[C(q, \dot{q})+K_{D}\right] s=Y_{2} K_{2} \tilde{\theta}
$$

where $\tilde{\theta}_{\mathbf{a}}:=\hat{\theta}_{\mathrm{a}}-\theta_{\mathrm{a}}^{*}$ is the augmented parameter crror.

Now, consider the Lyapunov function candidate

$$
2 \mathbf{V}=\mathbf{s}^{\top}[D(\mathbf{q})+\mathbf{J}] \mathbf{s}+\tilde{\mathbf{q}}^{\top} \mathbf{P} \tilde{\mathbf{q}}+\tilde{\theta}_{\mathbf{a}}^{\top} \Gamma_{\mathbf{a}}^{-1} \tilde{\theta}_{\mathbf{a}}
$$

where $\Gamma_{a}=\Gamma_{a}^{\top}>0$, and $P=P^{\top}>0$ is to be defined below. The derivative of (20) along the trajectories of (19) is 


$$
\dot{\mathbf{v}}=-\mathbf{s}^{\top} \mathbf{K}_{\mathrm{D}} \mathbf{s}+\tilde{\mathbf{q}}^{\top} \mathbf{P} \dot{\bar{q}}+\mathbf{s}^{\top} \mathbf{Y}_{\mathrm{a}} K_{\mathrm{a}} \tilde{\theta}_{\mathrm{a}}+\dot{\hat{\theta}}^{\top} \Gamma_{\mathrm{a}}^{-1} \tilde{\theta}_{\mathbf{a}}
$$

Then with the "gradient"adaptation law

$$
\dot{\hat{\theta}}_{\mathbf{a}}=-\left[\Gamma_{\mathbf{a}} K_{\mathrm{a}}\right] \mathbf{Y}_{\mathrm{a}}^{\top} \mathbf{s}
$$

we get

$$
\dot{\mathbf{v}}=-\mathbf{s}^{\top} K_{\mathrm{D}} \mathbf{s}+\tilde{\boldsymbol{q}}^{\top} \mathbf{p} \dot{\tilde{q}}
$$

Replacing $s$ by its expression (6) in (23) and choosing $P=2 \Lambda^{\top} K_{D}$, as in [9], one obtains

$$
\dot{\mathbf{V}}=-\dot{\tilde{q}}^{\top} K_{D} \dot{\tilde{q}}-\tilde{\mathbf{q}}^{\top} \Lambda^{\top} K_{D} \Lambda \tilde{\mathbf{q}}
$$

It is clear from (24) that $\dot{v}$ is a negative semidefinite function. So, invoking Lyapunov's direct method $|8|$ it can be shown that the trivial equilibrium of the closed loop (19), (17), (22), that is $\left[\tilde{q}^{\top}, \mathbf{s}^{\top}, \tilde{\theta}_{a}^{\top}\right]=0$ is globally stable in the sense of Lyapunov [8]. Hence, $\dot{\tilde{q}}$ and $\ddot{\tilde{q}}$ are also bounded implying that $\ddot{v}$ is bounded. Therefore, $\dot{v}$ is uniformly continuous and employing Barbalat's lemma it can be concluded that $\dot{\tilde{q}} \rightarrow 0$ and $\tilde{q} \rightarrow 0$ as $t \rightarrow \infty[8]$.

Since $1_{a}$, is a free positive definite symmetric matrix, one can define the gradient law gain as $I_{a k}:=I_{a} K_{a}$ and thus the gradient law (22) is replaced by

$$
\dot{\hat{\theta}}_{\mathbf{a}}=-\Gamma_{\text {ak }} \mathbf{Y}_{\mathbf{a}}^{\boldsymbol{T}} \mathbf{s}
$$

where

$$
I_{\text {ak }}=\operatorname{diag}\left[I_{1}\right]_{i=1, \ldots n}
$$

with arbitrary $\operatorname{rxr} I_{i}=l_{i}^{\top}>0$ since $K_{a}$ is of the special form described in (14). Then note that $\Gamma_{a}=\operatorname{diag}\left[k_{i}^{-1} \Gamma_{i}\right]_{l=1, n n}$. 
The complete adaptive motor voltage control is thus given by

$$
\begin{aligned}
& \mathbf{v}=\mathbf{Y}_{\mathrm{a}} \hat{\theta}_{\mathrm{a}}-\overline{\mathbf{K}}_{\mathrm{D}} \mathrm{s} \\
& \dot{\hat{\theta}}_{\mathrm{al}}=-1 ; y_{1} \mathbf{s}_{\mathrm{I}} \quad ; \quad \mathbf{i}=\mathbf{1}, . ., \mathrm{n}
\end{aligned}
$$

where $\bar{K}_{D}>0$ (diagonal) is a design matrix and can be chosen from lower bounds of the drive gains (see(11)).

\section{CONCLUSION}

In this paper a solution for the problem of designing a globally stable adaptive control for rigid robots with uncertain drive gains has been presented. The new control law is obtainod by means of a special overparametrization named "parameter unfolding". Global stability and convergence of tracking errors to zero are demonstrated.

\section{6 - REFERENCES}

[1] Corke, P.I. and B. Armstrong-Hélouvry. "A Search for Consensus Among Model Parameters Reported for the Puma 560 Robot", presented at the IEEE Robotics and Automation, San Diego, California, USA, vol.2, 1608-1613, May 1994.

[2] Guenther, R. and L. Hsu. "Variable Structure Adaptive Cascade Control of Rigid-Link Electrically-Driven Robot Manipulators", presented at the IEEE 32th CDC, San Antonio, Texas, USA, 2137-2142, December 1993.

[3] Hsu, L. and R. Guenther. "Variable Structure Adaptive Cascade Control of Multi-link Robot Manipulators with Flexible Joints: The Case of arbitrary uncertain flexibilities", presented at IEEE Robotics and Automation, Atlanta, USA, 340-345, May 1993.

[4] Khalil, W. and M. Gautier. "Computed Current Control of Robots", presented at the IFAC World Congress, Sydney, Australia, vol.4, 129-134, July 1993.

[5] Ortega,R. and M.W.Spong;"Adaptive Motion Control of Rigid Robots: a tutorial", presented at the IEEE 27th CDC, Austin, Texas, USA, 1988, 1575-1584.

[6] Reed, J.S. and P.A.loannou;"Instability Analysis and Robust Adaptive Control of Robotic Manipulators", presented at 27th IEEE CDC, Austin, Texas, USA, 1988, 1607-1612.

[7] Slotine, J.J.E and W.Li;"Adaptive Robot Control, a case study", IEEE Trans.Autom.Control, 33-11, November, 1988.

[8] Slotine, J.J.E. and W. Li. "Applied Nonlinear Control", Prentice-Hall International Editions, 1991.

[9] Spong, M.W., R. Ortega and R. Kelly. "Comments on "Adaptive Manipulator Control: A Case Study", IEEE Trans. Aut. Control, vol.35, no.6, 761-762, June 1990.

[10] Spong, M.W. and M. Vidyasagar. "Robot Dynamics and Control", John Wiley \& Sons, New York, 1989. 